\title{
SP1 promotes tumor angiogenesis and invasion by activating VEGF expression in an acquired trastuzumab-resistant ovarian cancer model
}

\author{
FENG SU $^{1,2^{*}}$, JING GENG $^{1 *}$, XINYING LI ${ }^{1}$, CHUAN QIAO ${ }^{1}$, LONGLONG LUO $^{1}$, \\ JIANNAN FENG ${ }^{1}$, XINJUN DONG ${ }^{1}$ and MING LV ${ }^{1}$
}
${ }^{1}$ Department of Molecular Immunology, Institute of Basic Medical Science, Academy of Military Medical Sciences, Haidian, Beijing 100850; ${ }^{2}$ Department of Medical Oncology, Affiliated Hospital of Binzhou Medical College, Binzhou, Shandong 256600, P.R. China

Received March 25, 2017; Accepted August 25, 2017

DOI: 10.3892/or.2017.5998

\begin{abstract}
Ovarian cancer is one of the most common gynecologic cancers and the leading cause of mortality in women worldwide. HER 2/neu is overexpressed in various types of cancers and is most commonly associated with decreased survival. Trastuzumab is a humanized anti-HER2 monoclonal antibody for the treatment of HER2-positive breast cancers. However, primary and/or acquired resistance occurs in up to $62 \%$ patients during the first year of treatment. Vascular endothelial growth factor (VEGF) is a well-known angiogenesis factor involved in many physiological and pathological processes. Its significance has been implicated in promoting tumor growth and metastasis via angiogenesis. In the present study, we demonstrated that the upregulation of SP1 enhanced expression of VEGF promoting the angiogenesis and migration of trastuzumab-resistant ovarian cancer cell line SKOV3-T. Our in vitro and in vivo results both gave evidence that the SP1-VEGF axis was responsible for the enhanced malignancy, angiogenesis and migration in the acquired trastuzumabresistant ovarian cancer cell model.
\end{abstract}

\section{Introduction}

Trastuzumab is a humanized anti-HER2 monoclonal antibody, which was approved for the treatment of HER2-positive

Correspondence to: Dr Xinjun Dong, Department of Medical Oncology, Affiliated Hospital of Binzhou Medical College, 522 Huanghe Road, Binzhou, Shandong 256600, P.R. China

E-mail: byfydxj@163.com

Dr Ming Lv, Department of Molecular Immunology, Institute of Basic Medical Science, Academy of Military Medical Sciences, 27 Taiping Road, Haidian, Beijing 100850, P.R. China

E-mail:1m62033@aliyun.com

*Contributed equally

Key words: ovarian cancer, trastuzumab resistance, angiogenesis, VEGF, SP1 cancers. Although trastuzumab is effective in various clinical cases, it eventually fails as primary and/or acquired resistance occurs in up to $62 \%$ of patients during the first year of treatment (1-3). For instance, many HER2/neu-positive cancers do not respond to trastuzumab treatment (de novo resistance), while many trastuzumab-responsive patients develop resistance after continuous trastuzumab infusion within 1 year (acquired resistance) (4-7).

Vascular endothelial growth factor (VEGF) is a valid proangiogenic factor that stimulates endothelial cell proliferation/growth, migration and increases vascular permeability (8). Its significance has been implicated in promoting solid tumor growth and metastasis via stimulating tumor-associated angiogenesis. Thus, blocking the activity of VEGF results in the starvation of tumors. Actually the function of VEGF in cancer is not limited to angiogenesis or vascular permeability as VEGF-mediated signaling also contributes to tumorigenesis, including the function of cancer stem cells and tumor initiation.

In our previous study, we induced an acquired trastuzumab resistance cell model SKOV3-T by long-term trastuzumab treatment of ovarian cancer cell line SKOV3 (9). In the present study, we found that the proliferation of SKOV3-T cells was much more rapid than that noted in SKOV3 both in vitro and in vivo. The microvessel counts were significantly higher in the SKOV3-T tumor tissues. The expression of VEGF was significantly upregulated in SKOV3-T. SP1, known as a VEGFupregulating molecule, was also found to be upregulated in SKOV3-T cells. The results suggest a possible SP1-VEGF axis which may be beneficial for angiogenesis and cell growth/ migration. Then, we explored the function of SP1 as well as downstream VEGF in resistant SKOV3-T cells using both in vitro assays. The results revealed that SP1 promoted tumor angiogenesis and invasion by activating VEGF expression in the acquired trastuzumab-resistant ovarian cancer model.

\section{Materials and methods}

Reagents. Trastuzumab (Herceptin ${ }^{\circledR}$ ) was obtained from F. Hoffmann-La Roche Ltd. (Shanghai, China). Antibodies 
of HIF- $\alpha$, STAT3, p-STAT3, P65, p-P65, SP1, histone H3, GAPDH and corresponding secondary antibodies were purchased from Cell Signaling Technology (Boston, MA, USA). Electrophoresis reagents and hybridization nitrocellulose filter membranes were obtained from Bio-Rad (Hercules, CA, USA). PE, DAPI, FITC and human VEGF-A Platinum ELISA kit were obtained from eBioscience (San Diego, CA, USA). Goat anti-human CD31 antibody was obtained from Abcam Biotechnology (Cambridge, MA, USA). BCA protein assay kit and enhanced chemiluminescent (ECL) reagents were purchased from Pierce (Rockford, IL, USA). Cell culture medium Dulbecco's modified Eagle's medium (DMEM) and fetal bovine serum (FBS) were purchased from HyClone (Logan, UT, USA). SP1 interference plasmids, SP1 shRNAs (1-4), were purchased from GeneChem (Shanghai, China). Female 6-week-old BALB/c nude mice were purchased from the Vital River Laboratory (Beijing, China). TransiT2020 transfection reagent was purchased from Mirus Bio LLC (Madison, WI, USA). Transwell chamber was obtained from Merck Millipore (Darmstadt, Germany). All other chemicals were obtained from commercial sources of analytical grade.

Cell culture. Human ovarian cancer cell line SKOV3 was obtained from the American Type Culture Collection (ATCC; no. HTB-77) (Manassas, VA, USA). Acquired trastuzumabresistant ovarian cancer cell line SKOV3-T was developed by continuously culturing SKOV3 cells in the presence of $20 \mu \mathrm{g} / \mathrm{ml}$ trastuzumab as previously described (9). SKOV3-T cells were maintained in the presence of $10 \mu \mathrm{g} / \mathrm{ml}$ trastuzumab (9). SKOV3 and SKOV3-T cells were cultured in DMEM supplemented with $10 \%$ heat-inactivated FBS and $100 \mathrm{U} / \mathrm{ml}$ penicillin and streptomycin. Cells were cultured at $37^{\circ} \mathrm{C}$ in $5 \% \mathrm{CO}_{2}$. Human umbilical vein endothelial cells (HUVECs) were obtained from human umbilical veins as previously described (10).

HUVEC proliferation assay. HUVECs were suspended at a density of $1 \times 10^{5} / \mathrm{ml}$ and were seeded in a 96-well plate (100 $\mu \mathrm{l} /$ well). After serum-free starvation overnight, the cells were treated with 4 or 8 times diluted cell culture supernatant of SKOV3 or SKOV3-T cells. After cultivation for $10 \mathrm{~h}$ at $37^{\circ} \mathrm{C}, 10 \mu \mathrm{l} /$ well of Cell Counting Kit-8 (CCK8; Dojindo Laboratories, Kumamoto, Japan) was added, and the plate was incubated for another $4 \mathrm{~h}$. The absorbance was measured using a spectrophotometer at $450 \mathrm{~nm}$ to determine the cell viability.

Immunohistochemistry $(I H C)$. A week after the last observation, mice were sacrificed, and the tumors were separated and fixed with $10 \%$ formaldehyde. Paraffin-embedded tissue sections were processed, deparaffinized, rehydrated and quenched for endogenous peroxidase activity. Sections were stained with anti-CD31 antibody (dilution 1:100), and then incubated with horseradish peroxidase-conjugated secondary antibody. Finally, the sections were developed with diaminobenzidine and counterstained with hematoxylin. Images were captured using an Olympus BX5 microscope with an UPlanFL N digital camera (10x0.13 numeric aperture objective). Any single brown-stained cell or cluster of endothelial cells that was clearly separated from adjacent microvessels, tumor cells and other connective tissue elements was considered a vessel.
The number of CD31-positive capillaries was counted from 5 randomly chosen fields.

Transwell assay. The migration and invasion capacity of the SKOV3-T and SKOV3 cells was quantified by Transwell assays using a permeable membrane system plate with $8-\mu \mathrm{m}$ pore size (Corning Costar; Corning, Inc., Corning, NY, USA). SKOV3-T and SKOV3 cells were starved in serum-free DMEM overnight and resuspended in DMEM at a density of $4 \times 10^{5} / \mathrm{ml}$. Then, $250 \mu \mathrm{l}$ cells were seeded in the top chambers; meanwhile, $750 \mu 1 \mathrm{FBS}$ was added to the bottom chamber. The cells were induced to migrate towards medium containing 0 , 10 or $100 \mu \mathrm{g} / \mathrm{ml}$ Avastin. After 6, 12 or $24 \mathrm{~h}$ of incubation at $37^{\circ} \mathrm{C}$, non-migrated cells on top of the upper membrane were removed thoroughly with cotton swabs. The migrated cells below the membrane were fixed with $4 \%$ paraformaldehyde, stained with Giemsa solution, and then analyzed by a bright-field microscopy. Cell images were captured using an Olympus BX5 microscope with an UPlanFL N digital camera (10x0.13 numeric aperture objective). The number of migrated cells was counted from 5 randomly chosen fields.

SP1 silencing in SKOV3-T cells. SP1-silencing shRNA plasmids (1-4) were used to knock down SP1 expression in the SKOV3-T cells. SKOV3-T cells $\left(2.5 \times 10^{5}\right.$ cells/well) were seeded into a 6-well plate and cultured overnight. SP1-silencing shRNA plasmid $(2.5 \mu \mathrm{g})$ and $7.5 \mu \mathrm{l}$ TransIT-2020 transfection reagent were added into $250 \mu \mathrm{l}$ Opti-MEM I reduced-serum medium, mixed well and the mixture was placed at room temperature (RT) for 15-30 min. Then, the TransIT-2020 reagent-DNA complexes were added to the cell supernatant for $72 \mathrm{~h}$ of incubation. The transfectants were treated using $12 \mu \mathrm{g} / \mathrm{ml}$ puromycin (Sigma-Aldrich, St. Louis, MO, USA) for 7 days to obtain SP1 silenced clones.

Western blotting. SKOV3 cells were seeded into 6-well plates at a density of $5 \times 10^{5}$ cells $/ \mathrm{ml}$. Meanwhile, SKOV3-T cells were seeded into 6-well plates at a density of $3 \times 10^{5}$ cells $/ \mathrm{ml}$. After being serum-starved overnight, cells were collected and the supernatant of the cell lysate was subjected to SDS-PAGE analysis using $10 \%$ gel. Samples were transferred from the gel onto a nitrocellulose membrane. The membrane was blocked with non-fat $5 \%$ milk for $1 \mathrm{~h}$ at RT, and then incubated with anti-HIF- $\alpha$, STAT3, p-STAT3, P65, p-P65, SP1, GAPDH and histone $\mathrm{H} 3$ antibodies (1:1,000 dilution) overnight at $4{ }^{\circ} \mathrm{C}$, respectively. After washes with Tris-buffered saline with Tween-20 (TBST) 3 times, the membranes were incubated with the appropriate secondary antibodies conjugated with HRP (dilution 1:2,500) for $1 \mathrm{~h}$ at RT. Signals on the membrane were detected and visualized by enhanced chemilunimescence (ECL) detection system and autoradiography. Relative protein expression in whole cells or the cytoplasm was normalized to GAPDH intensity and SP1 expression in the nucleus was normalized to $\mathrm{H} 3$ intensity.

$R T-P C R$. Total RNA was isolated from the cells using a total RNA isolation kit (RNAgents; Promega, Madison, WI, USA). The total RNA $(1.0 \mu \mathrm{g})$ was reverse transcribed using an oligo(dT) 18-mer and reverse transcription system (Promega). PCR was carried out using selective primers for human VEGF 
(sense primer, 5'-GCTACTGCCATCCAATCGAG-3' and antisense primer, 5'-TGCATTCACATTTGTTGTGC-3'); and GAPDH (sense primer, 5'-TCCAAGGGTCCGCTGCAGTC-3' and antisense primer, 5'-CGTTCACCTTGATGAGCCCACG TTCACCTTGATGAGCCCATT-3'). PCR was carried out for 35 cycles under the following conditions: denaturation at $95^{\circ} \mathrm{C}$ for $30 \mathrm{sec}$, annealing at $58^{\circ} \mathrm{C}$ for $30 \mathrm{sec}$, and elongation at $72^{\circ} \mathrm{C}$ for $30 \mathrm{sec}$.

Xenograft mouse model. Six-week-old female BALB/c nude mice were purchased from Vital River Laboratory, housed in a barrier environment with 12:12 h light-dark cycle, and fed normal mouse laboratory diet. Thirty-two mice were divided randomly into 4 groups $(n=8)$. Each mouse was subcutaneously inoculated on the back with $2 \times 10^{6} / 0.1 \mathrm{ml} \mathrm{SKOV3-T,}$ SKOV3, 1F10 or 3D7 cells on day 0. Mice were observed twice a week and the perpendicular tumor dimensions were measured using a Vernier scale caliper. The tumor volumes were calculated according to the following equation: Tumor volume $\left(\mathrm{mm}^{3}\right)=1 / 2 \times$ (length) $\mathrm{x}$ (width $)^{2}$. A week after the last observation, the mice were sacrificed and the tumors were separated for IHC analysis. All procedures were administered in strict agreement with international guidelines for the care and use of laboratory animals and approved by the Animal Ethics Committee of the Institute of Basic Medical Sciences, Academy of Military Medical Sciences, Beijing.

Enzyme-linked immunosorbent assay (ELISA). First, we prepared all reagents and standard samples following the instructions of the human VEGF ELISA kit. ELISA plates were blocked, diluted cultured media were added and antibody was detected following $2 \mathrm{~h}$ of incubation at $37^{\circ} \mathrm{C}$. After washing, horseradish peroxidase-conjugated goat anti-human IgG was added for $45 \mathrm{~min}$ of incubation at RT. The peroxidase reaction was developed with substrate solution provided in the ELISA kit and the light absorbance was measured with an ELISA reader at $450 \mathrm{~nm}$.

Confocal microscopy. Cell monolayers were washed with phosphate-buffered saline (PBS) before being fixed with $4 \%$ paraformaldehyde for $10 \mathrm{~min}$. After washing with PBS, the samples were permeabilized with $0.25 \%$ Triton-PBS for $8 \mathrm{~min}$ at RT. The culture dishes were blocked with $10 \%$ FBS for $1 \mathrm{~h}$ at RT, and then incubated with various antibodies (dilution $1: 100)$ overnight at $4^{\circ} \mathrm{C}$. Then, the samples were incubated with secondary antibody with fluorescence (PE, DAPI) for $30 \mathrm{~min}$ at RT. Finally, samples were washed and visualized by laser scanning confocal microscope (LSCM).

Statistical analysis. Data are expressed as the mean \pm SD. Pairwise differences between several groups were compared. Significant differences were analyzed by the one-way analysis of variance (ANOVA), and P-values of $<0.05$ were taken as statistically significant.

\section{Results}

Herceptin-resistant SKOV3-T cells possess enhanced tumorigenesis and angiogenesis. HER2/neu-positive SKOV3 cells were continuously cultured in the presence of $20 \mu \mathrm{g} / \mathrm{ml}$ trastuzumab for 8 months, resulting in trastuzumab-resistant SKOV3-T cells. The xenograft model results revealed that the in vivo tumor growth rate of SKOV3-T was significantly more rapid than that of the SKOV3 cells. Meanwhile, the shape of SKOV3-T xenografts seemed more irregular than SKOV3. The results suggested the possibility of enhanced migration of SKOV3-T cells (Fig. 1A; $\mathrm{P}<0.001$ ). Tumor sections were then stained with anti-CD31 (a vascular endothelial marker) antibody for IHC assay. As shown in Fig. 1B, a significant increase in microvessel density was observed in the xenograft tumors of SKOV3-T cells, which may facilitate the faster growth of SKOV3-T xenografts. Therefore, we assumed that trastuzumab treatment may induce the upregulation of angiogenesis factors in SKOV3-T cells in order to accelerate vessel formation and tumor growth in vivo.

Overexpression of VEGF in SKOV3-T cells accelerates the migration of SKOV3-T cells. VEGF is the most important proangiogenic factor, which enhances the proliferation of endothelial cells. As shown in Fig. 2A, the proliferation of HUVECs cultured in the SKOV3-T supernatant was markedly increased, suggesting higher VEGF secretion in the supernatant of SKOV3-T cells $(\mathrm{P}<0.01)$. ELISA assay results confirmed the VEGF overexpression in the SKOV3-T cells, which was nearly 3 times higher than that in the SKOV3 cells (Fig. 2B). RT-PCR analysis results (Fig. 2C) were consistent with the protein detection results; VEGF was overexpressed in the SKOV3-T when compared to the expression noted in the SKOV3 cells.

Endothelial cell migration is the essential/functional process in angiogenesis. Thus, a Transwell assay was employed to evaluate the difference in cell migration between the two cell lines. As shown in Fig. 2D, SKOV3-T cells exhibited a markedly stronger migration ability $(\mathrm{P}<0.001)$. The number of migratory SKOV3-T cells was $>6$ times that of the SKOV3 cells after 6 or $24 \mathrm{~h}$. Meanwhile, along with the increased concentration of Avastin, the migration capacity of the SKOV3-T cells was inhibited in a dose-dependent manner (Fig. 2E). These results indicate that the enhanced migration activity of the SKOV3-T cells was induced by the overexpression of VEGF, which also explained why this cell line proliferated much faster than SKOV3 as well as had enhanced angiogenesis.

SP1 promotes VEGF overexpression in SKOV3-T cells. The 5 -flanking region of the VEGF gene contains the binding sites of several transcription factors, which play an important role in regulating the transcription of the VEGF gene. Western blot results revealed that SP1 expression was upregulated, while the levels of other transcription factors were identical to those in the SKOV3 cells (Fig. 3A). SP1 seemed to respond to VEGF overexpression in the trastuzumab-resistant ovarian cancer SKOV3-T cells. Furthermore, the SP1 level in the cytoplasm appeared to be identical between the SKOV3-T and SKOV3 cells (Fig. 3B, left panel; $\mathrm{P}<0.01$ ), and the level of SP1 localized in the nucleus of SKOV3-T cells was much higher, suggesting its possible function to enhance the transcription level of VEGF (Fig. 3B, right panel; $\mathrm{P}<0.01$ ). Confocal microscopy observation also showed the similar consequence of SP1 overexpression in SKOV3-T cells, and we observed more SP1 gathered in the nucleus in contrast to that observed in the SKOV3 cells (Fig. 3C). 

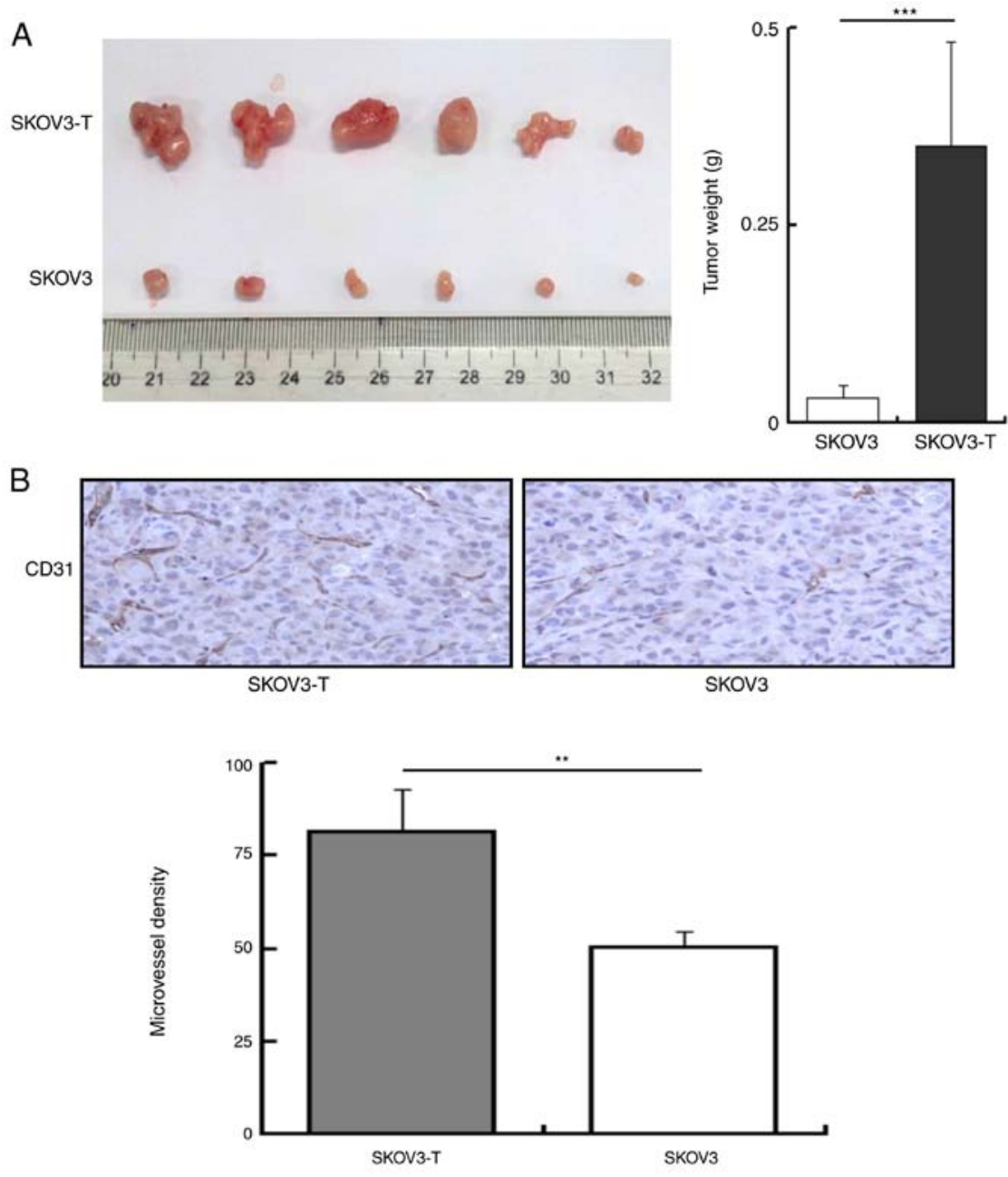

Figure 1. In vivo tumorigenesis and angiogenesis are enhanced in SKOV3-T cells. (A) SKOV3-T xenografts were obviously larger than those derived from SKOV3, while the shape was irregular to suggest possible enhanced angiogenesis $\left({ }^{* * *} \mathrm{P}<0.01\right)$. (B) The microvessel density of SKOV3-T xenografts was higher than that of SKOV3 tumor specimens $\left({ }^{* *} \mathrm{P}<0.01\right)$. Female nude mice were subcutaneously inoculated with SKOV3 or SKOV3-T cells $\left(2 \times 10^{6}\right)$ into the fat pad at day 0 . The tumors were observed twice a week for 14 days. On day 21 , tumors were separated and analyzed.

A

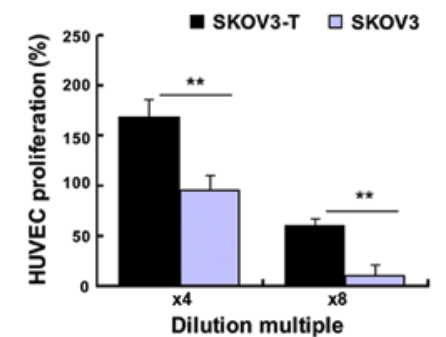

D

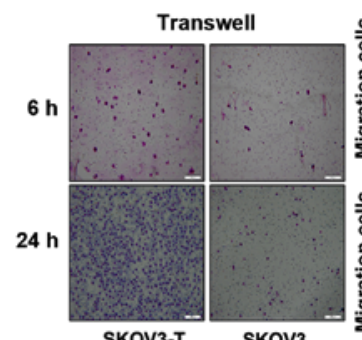

SKOV3-T
B

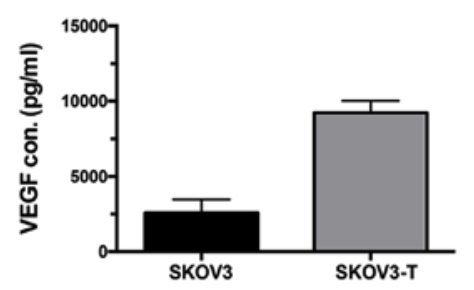

E

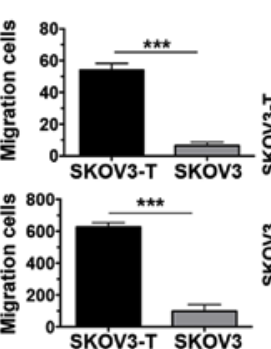

C
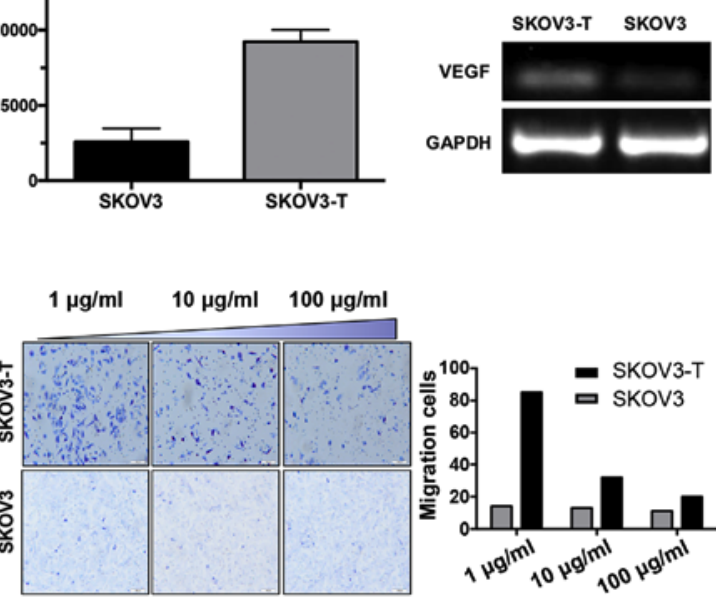

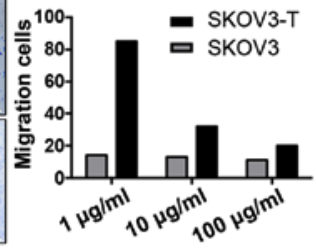

Figure 2. VEGF is upregulated in SKOV3-T cells, and anti-VEGF antibody (Avastin) inhibits the enhanced migration activity of SKOV3-T cells. (A) HUVEC proliferation was enhanced by SKOV3-T cell supernatant; ** P<0.01. (B) VEGF secretion in SKOV3-T cells was higher than that in SKOV3 cells. (C) VEGF expression in SKOV3-T cells was also higher than that in SKOV3 cells. (D) The migration ability of SKOV3-T cells was higher than that noted in the SKOV3 cells; ${ }^{* * *} \mathrm{P}<0.001$. (E) Avastin inhibited the migration ability of the SKOV3-T cells in a dose-dependent manner, which suggests that the enhanced migration of SKOV3-T was induced at least partially by upregulation of VEGF. 
A

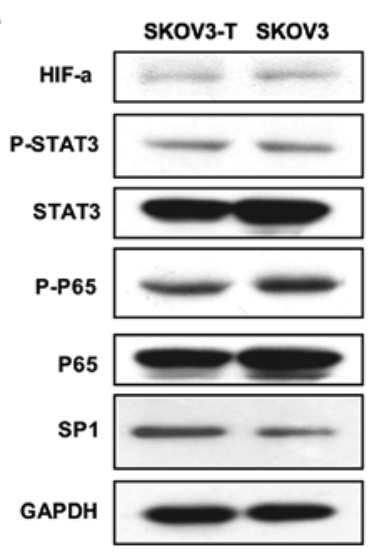

B
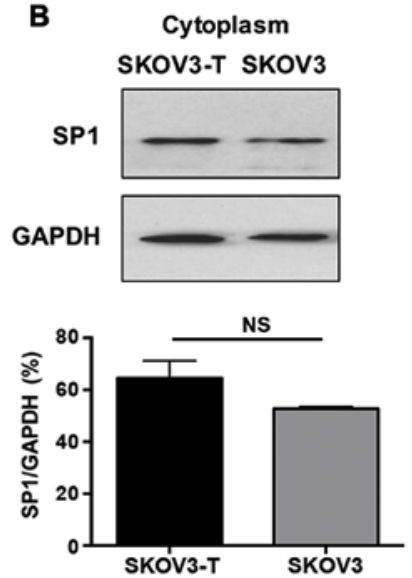

DAPI
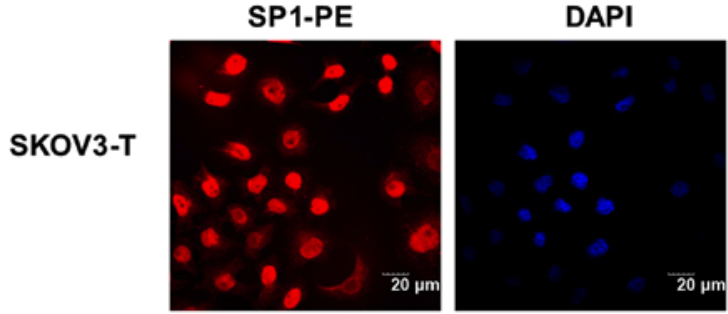

$20 \mu$
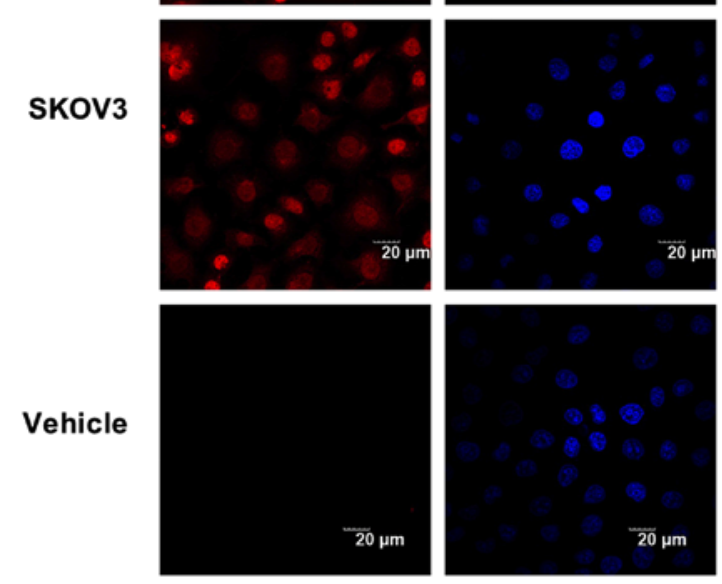
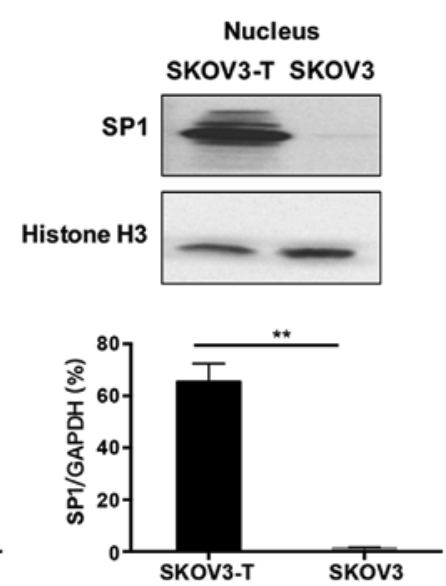

C
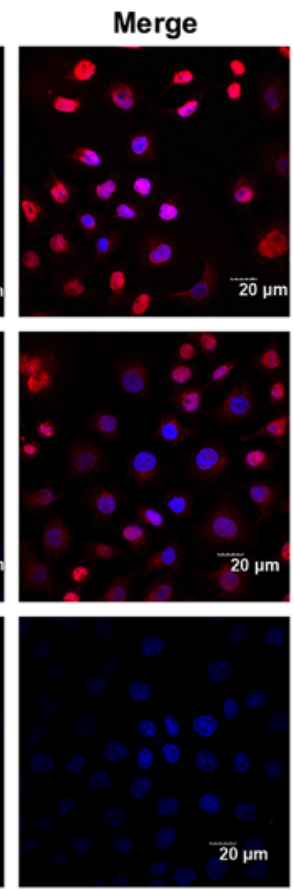

Figure 3. Upregulation of SP1 in the nucleus may be the key transcriptional factor to induce the overexpression of VEGF in SKOV3-T cells. (A) Expression of possible VEGF-associated transcriptional factors in SKOV3-T and SKOV3 cells, while SP1 level was different. (B) SP1 expression in the cytoplasm (left) and nucleus (right) of SKOV3-T and SKOV3, respectively. NS, not significant. It is suggested that higher SP1 in the nucleus may influence the transcription of VEGF. (C) Confocal microscopy observation of enhanced SP1 distribution in SKOV3-T cells. Scale bar, $25 \mu \mathrm{m}$.

SPl knockdown inhibits VEGF-mediated migration in SKOV3-T cells. In order to evaluate and confirm whether SP1 influences VEGF expression as well as VEGF-induced migration in SKOV3-T cells, we synthesized SP1 shRNAs (1-4) and transfected the SKOV3-T cells, respectively, to knock down SP1 expression. Then, the cells were collected for western blot analysis to confirm the markedly reduced SP1 (Fig. 4A). Along with SP1 knockdown, the VEGF expression level in the supernatant was lower in the SKOV3-T cells, indicating the importance of SP1 to upregulate VEGF (Fig. 4B; $\mathrm{P}<0.01$ ); Furthermore, SP1 knockdown by SP1 shRNA inhibited the migration capacity of the SKOV3-T cells, which confirmed the presence of an 'SP1-VEGF axis' to induce enhanced migration following long-time trastuzumab treatment of SKOV3 cells (Fig. 4C; $\mathrm{P}<0.01$ ).

After subcloning, we screened two SP1-knockdown SKOV3-T cell clones, named 3D7 and 1F10, for next-step in vivo assays. As shown in Fig. 4D, the SP1 expression in the 3D7 and 1F10 cells was low. The clone 1F10 had a lower expression of SP1 compared with 3D7, thus 3D7 was set as a 'partially knocked down' clone with medium SP1 expression level between SKOV3-T and SKOV3 cells (Fig. 4D). The difference between the two clones in regards to VEGF secretion was detected by ELISA. The results revealed that the concentration of VEGF was higher in 1F10 than that in the 3D7 cell line (Fig. 4E; $\mathrm{P}<0.01, \mathrm{P}<0.001$ ).

SP1-knockdown inhibits the tumor growth and angiogenesis of SKOV3-T cells in vivo. Six-weeks old female nude mice were randomly divided into 4 groups with 8 mice/group $(n=8)$, and were subcutaneously inoculated with SKOV3-T, SKOV3, 1F10 or $3 \mathrm{D} 7$ cells $\left(2 \times 10^{6} / \mathrm{mice}\right)$ and the xenografts were allowed to grow for 28 days. The tumor size was measured twice a week. As shown in Fig. 5A-C, the mean tumor size/weight of 
A

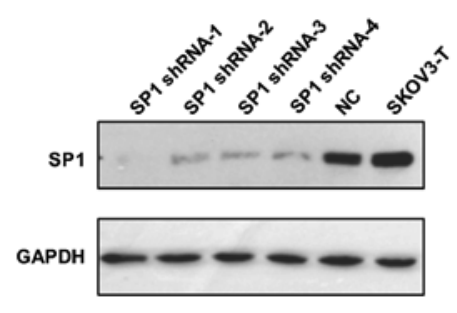

C
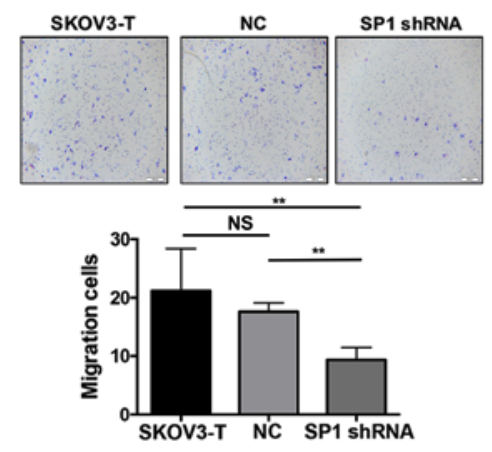

B

D
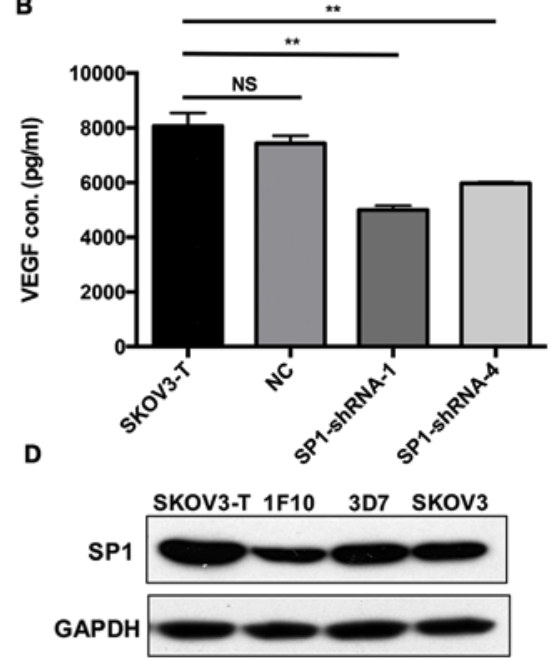

E

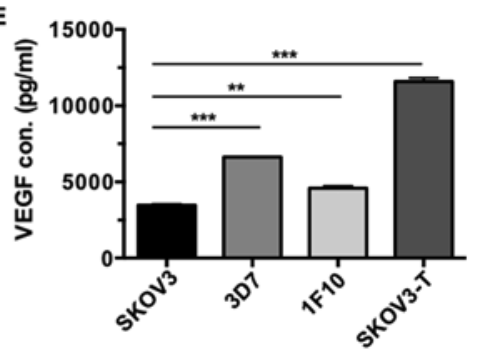

Figure 4. SP1 promotes VEGF-related migration in SKOV3-T cells. (A) Western blot analysis of SP1 expression in SKOV3-T cells transfected with SP1knockdown shRNA 1-4 or control shRNA, respectively. (B) VEGF expression in the supernatant ofSKOV3-T cells was lower due to SP1-knockdown, suggesting the importance of SP1in VEGF overexpression; ** P<0.01. NS, not significant. (C) VEGF-induced migration of SKOV3-T cells was inhibited by SP1-knockdown ${ }^{* *} \mathrm{P}<0.01$. NS, not significant. (D) Western blot analysis of SP1 in SP1-knockdown cell clones. (E) The VEGF concentration in the SP1-knockdown cell clones (3D7 and 1F10) was obviously decreased in contrast to that in the SKOV3-T cells. ${ }^{* *} \mathrm{P}<0.01,{ }^{* * *} \mathrm{P}<0.001$.

the $1 \mathrm{~F} 10$ - and 3D7-derived tumors were significantly smaller than the tumors derived from the SKOV3-T cells, indicating that SP1-knockdown reversed trastuzumab-resistance to inhibit the carcinogenicity of the SKOV3-T cells. However, the 'partial knockdown' clone 3D7 xenografts were slightly larger than 1F10, while 1F10 tumors were similar to those derived from the SKOV3 cells, which was consistent with the in vitro assay trend. Furthermore, the microvessel density assay showed that SP1 knockdown inhibited the angiogenesis of tumors, confirming the importance of the SP1-VEGF axis in trastuzumab-resistant SKOV3-T cells (Fig. 5D).

\section{Discussion}

Ovarian cancer is the fourth common cancer in terms of mortality among women, and is the leading cause of death from gynecologic malignancies worldwide $(11,12)$. HER2 is overexpressed in $\sim 25-30 \%$ of ovarian cancer patients, yet anti-HER 2 therapies display limited clinical response in the treatment of HER2-positive ovarian cancer (13). It has been reported that many HER2/neu-positive cancer patients indeed do not respond to trastuzumab treatment (de novo resistance), or develop resistance after continuous trastuzumab infusion within 1 year (acquired resistance) (4-7), which may be the reason why trastuzumab therapy for ovarian cancer patients usually fails in clinical trials.

Previously, we established in vitro an acquired trastuzumab-resistant ovarian cancer cell model, SKOV3-T, as previously described (9). SKOV3-T cells possessed enhanced proliferation and carcinogenesis characteristic in vivo (Fig. 1A). We found overexpression of IGF-1R and HER3 in the resistant cells (9). However, IGF-1R and HER3 were not the key overexpressed biomarkers of the resistant cell model. Anti-IGF-1R treatment only partially inhibited the cell growth, and the treated cells maintained rapid growth either in vitro or in vivo; therefore it failed to reverse the trastuzumab resistance thoroughly (14).

Recently, we noted the enhanced angiogenesis and migration characteristics in the in vivo xenograft assays, by which we inferred the overexpression of VEGF in SKOV3-T cells. It was reported that ovarian tumors usually contain a rich vascular network and are highly dependent on VEGFmediated angiogenesis (15). Angiogenesis is the progression of new blood vessel formation, which plays a key role in physiological and pathological processes $(16,17)$. VEGF plays a pivotal role in human tumorigenesis and angiogenesis of cancer. In the present study, as shown in Fig. 1B, the SKOV3-T xenograft tumors contained more microvessels, as the density was higher than SKOV3. The HUVEC-based cell proliferation assay indicated the presence of higher VEGF in the SKOV3-T cell culture (Fig. 2A). ELISA and RT-PCR also provided identical data (Fig. 2B and C). At the same time, we also noted that the edges of the SKOV3-T tumors were markedly more irregular, suggesting possible enhanced migration activity of the SKOV3-T cells probably due to the overexpression of VEGF. A Transwell assay was used to confirm the assumption 
A

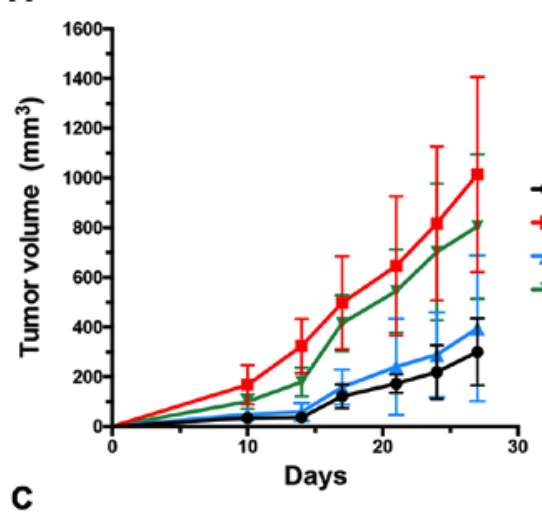

C

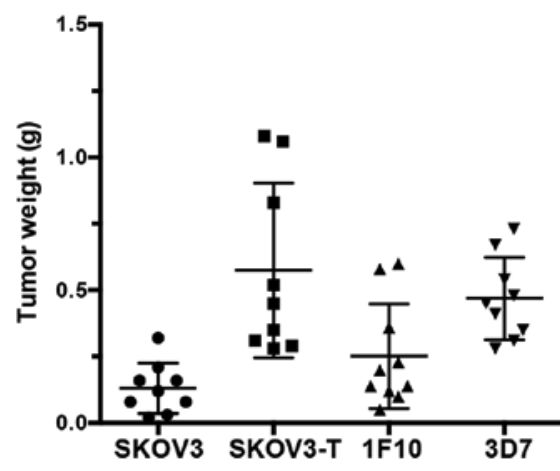

B
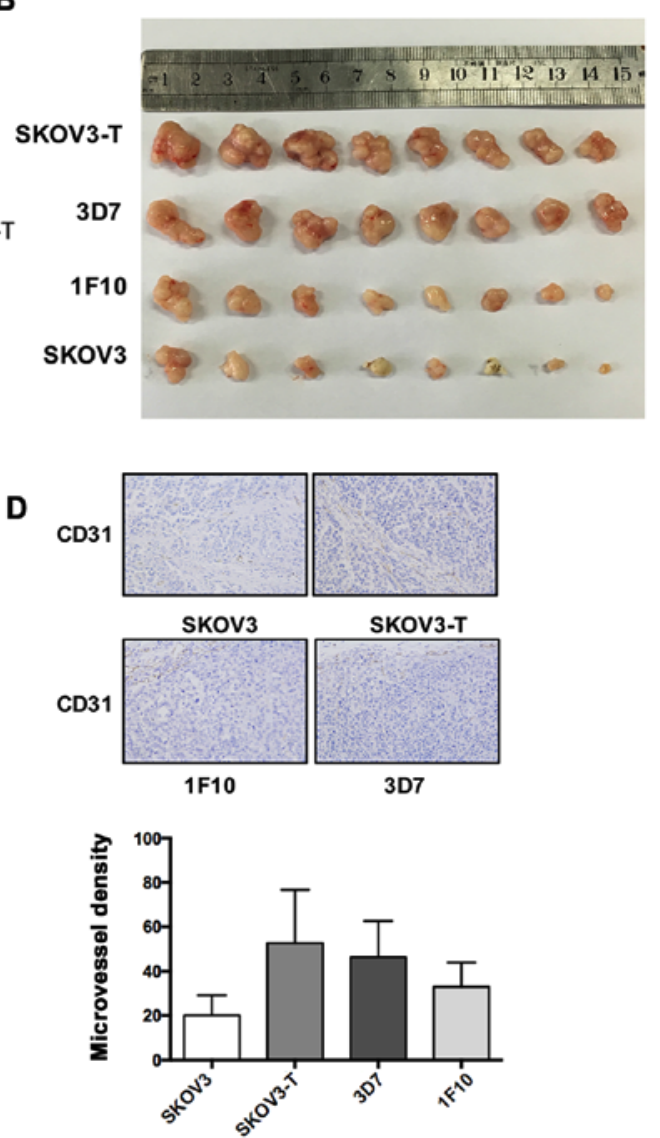

Figure 5. SP1-knockdown inhibits the tumor growth and angiogenesis of SKOV3-T cells in vivo. (A) Average volume of SKOV3-T cell-derived tumors was larger than SP1-knockdown xenografts. (B) Tumor image of the xenografts. (C) Tumor weight of the xenografts, showing that the SP1-VEGF axis was key to controlling the tumor growth in vivo. (D) Microvessel density of SP1-knockdown xenografts was also lower than SKOV3-T, showing that SP1 may be a good target with which to kill trastuzumab-resistant ovarian cancer cells (SKOV3).

concerning cell migration (Fig. 2D), and anti-VEGF antibody, Avastin, showed specific inhibitory function against migration in a dose-dependent manner (Fig. 2E), suggesting the importance of higher VEGF to induce stronger migration activity in the SKOV3-T cells.

Furthermore, we analyzed the possible translational factors of VEGF, e.g. HIF-1 $\alpha$, STAT3, P65 and SP1. According to western blotting of whole cells (Fig. 3A), SP1 was upregulated, particularly in the nuclei of SKOV3-T cells (Fig. 3B and C). $\mathrm{Sp} 1$ is well-known as a transcription factor which binds to the promoters of many target genes and is implicated in the regulation of multiple essential biological processes, including cell apoptosis and growth, cell cycle progression, angiogenesis and metastasis (18-20). Sp1 not only regulates the expression of multiple genes, but also the Sp1 gene itself. Sp1 levels often correlate with tumor stage and poor prognosis (21). Constitutive activation of SP1 plays a critical role in VEGF expression $(22,23)$. SP1 was found to enhance cell invasion by upregulating VEGF in lung cancer and LKB1, an inhibitor of SP1, suppressed the invasion (22). In colon cancers, SP1 was confirmed as a predominant factor required for AKT-mediated induction of VEGF (24). The self-renewal ability, drug resistance, and metastasis potential of colon CSCs may be partially due to preferentially high expression of SP1 (24).

In the present study, we used shRNAs to knock down SP1 (Fig. 4A) to identify the function of the SP1-VEGF axis in cell migration and angiogenesis of SKOV3-T cells, which was found to downregulate the corresponding VEGF expression in SKOV3-T cells (Fig. 4B) and inhibit VEGF-induced migration in vitro (Fig. 4C). Then, we screened two SP1-knockdown clones, 3D7 and 1F10, in which 3D7 was set as a "partial knockdown' clone (Fig. 4D). Similar to the trend in SP1 expression, the VEGF secretion in the two clones was decreased; more importantly, VEGF in the 1F10 supernatant was lower than that in 3D7 (Fig. 4D), indicating that SP1 was pivotal in the regulation of migration and VEGF, and influenced malignancy and enhanced the angiogenesis of tumors.

In vivo experiments also confirmed SP1 as the core factor to control VEGF-related tumor growth and microvessel formation. Notably, the average tumor size of the 1F10 cellderived xenografts was almost as small as those derived from the SKOV3 cells, indicating that SP1 knockdown successfully inhibited the growth of trastuzumab-resistant SKOV3-T xenografts (Fig. 5B). Meanwhile, IHC analysis showed reduced microvessel density in the $1 \mathrm{~F} 10$ tumors which showed that SP1 knockdown inhibited VEGF-dependent angiogenesis, and therefore, reversed the trastuzumab resistance in the ovarian cancer cells (Fig. 5D). Although further investigation is warranted to understand the related mechanism, the in vitro and in vivo data provide evidence of the SP1-VEGF axis that induced malignancy, angiogenesis and migration in the acquired trastuzumab-resistant ovarian cancer cell model. 


\section{Acknowledgements}

The present study was supported by the National Natural Science Foundation of China (no. 81272528).

\section{References}

1. Esteva FJ, Yu D, Hung MC and Hortobagyi GN: Molecular predictors of response to trastuzumab and lapatinib in breast cancer. Nat Rev Clin Oncol 7: 98-107, 2010.

2. Madrid-Paredes A, Cañadas-Garre M, Sánchez-Pozo A and Calleja-Hernández MA: Non-HER2 signaling pathways activated in resistance to anti-HER2 therapy in breast cancer. Breast Cancer Res Treat 153: 493-505, 2015.

3. Madrid-Paredes A, Cañadas-Garre M, Sánchez-Pozo A and Calleja-Hernández MA: De novo resistance biomarkers to anti-HER2 therapies in HER2-positive breast cancer. Pharmacogenomics 16: 1411-1426, 2015.

4. Kruser TJ and Wheeler DL: Mechanisms of resistance to HER family targeting antibodies. Exp Cell Res 316: 1083-1100, 2010.

5. Fiszman GL and Jasnis MA: Molecular mechanisms of trastuzumab resistance in HER 2 overexpressing breast cancer. Int J Breast Cancer 2011: 352182, 2011.

6. Köninki K, Barok M, Tanner M, Staff S, Pitkänen J, Hemmilä P, Ilvesaro J and Isola J: Multiple molecular mechanisms underlying trastuzumab and lapatinib resistance in JIMT-1 breast cancer cells. Cancer Lett 294: 211-219, 2010.

7. Nahta R and Esteva FJ: HER2 therapy: Molecular mechanisms of trastuzumab resistance. Breast Cancer Res 8: 215, 2006

8. Ferrara N, Gerber HP and LeCouter J: The biology of VEGF and its receptors. Nat Med 9: 669-676, 2003.

9. Jia Y, Zhang Y, Qiao C, Liu G, Zhao Q, Zhou T, Chen G, Li Y, Feng J, Li Y, et al: IGF-1R and ErbB3/HER3 contribute to enhanced proliferation and carcinogenesis in trastuzumabresistant ovarian cancer model. Biochem Biophys Res Commun 436: 740-745, 2013.

10. Yang J, Wang Q, Qiao C, Lin Z, Li X, Huang Y, Zhou T, Li Y, Shen B, Lv M, et al: Potent anti-angiogenesis and anti-tumor activity of a novel human anti-VEGF antibody, MIL60. Cell Mol Immunol 11: 285-293, 2014.

11. Nusrat O, Belotte J, Fletcher NM, Memaj I, Saed MG, Diamond MP and Saed GM: The role of angiogenesis in the persistence of chemoresistance in epithelial ovarian cancer. Reprod Sci 23: 1484-1492, 2016.

12. Thibault B, Castells M, Delord JP and Couderc B: Ovarian cancer microenvironment: Implications for cancer dissemination and chemoresistance acquisition. Cancer Metastasis Rev 33: 17-39, 2014.
13. Bookman MA, Darcy KM, Clarke-Pearson D, Boothby RA and Horowitz IR: Evaluation of monoclonal humanized anti-HER2 antibody, trastuzumab, in patients with recurrent or refractory ovarian or primary peritoneal carcinoma with overexpression of HER2: A phase II trial of the Gynecologic Oncology Group. J Clin Oncol 21: 283-290, 2003.

14. Wang W, Zhang Y, Lv M, Feng J, Peng H, Geng J, Lin Z, Zhou T, Li X, Shen B, et al: Anti-IGF-1R monoclonal antibody inhibits the carcinogenicity activity of acquired trastuzumab-resistant SKOV3. J Ovarian Res 7: 103, 2014.

15. Ramakrishnan S, Subramanian IV, Yokoyama Y and Geller M: Angiogenesis in normal and neoplastic ovaries. Angiogenesis 8: $169-182,2005$.

16. Folkman J: Angiogenesis in cancer, vascular, rheumatoid and other disease. Nat Med 1: 27-31, 1995

17. Bergers $\mathrm{G}$ and Benjamin LE: Tumorigenesis and the angiogenic switch. Nat Rev Cancer 3: 401-410, 2003.

18. Chen Y, Huang Y, Huang Y, Xia X, Zhang J, Zhou Y, Tan Y, He S, Qiang F, Li A, et al: JWA suppresses tumor angiogenesis via Spl-activated matrix metalloproteinase- 2 and its prognostic significance in human gastric cancer. Carcinogenesis 35: 442-451, 2014.

19. Hsu TI, Wang MC, Chen SY, Yeh YM, Su WC, Chang WC and Hung JJ: Spl expression regulates lung tumor progression. Oncogene 31: 3973-3988, 2012

20. Jungert K, Buck A, von Wichert G, Adler G, König A, Buchholz M, Gress TM and Ellenrieder V: Sp1 is required for transforming growth factor-beta-induced mesenchymal transition and migration in pancreatic cancer cells. Cancer Res 67: 1563-1570, 2007.

21. Vizcaíno C, Mansilla S and Portugal J: Spl transcription factor: A long-standing target in cancer chemotherapy. Pharmacol Ther 152: 111-124, 2015.

22. Liang X, Li ZL, Jiang LL, Guo QQ, Liu MJ and Nan KJ: Suppression of lung cancer cell invasion by LKB1 is due to the downregulation of tissue factor and vascular endothelial growth factor, partly dependent on SP1. Int J Oncol 44: 1989-1997, 2014.

23. Hirose T and Horvitz HR: An Sp1 transcription factor coordinates caspase-dependent and -independent apoptotic pathways. Nature 500: 354-358, 2013.

24. Zhao Y, Zhang W, Guo Z, Ma F, Wu Y, Bai Y, Gong W, Chen Y, Cheng T, Zhi F, et al: Inhibition of the transcription factor Sp1 suppresses colon cancer stem cell growth and induces apoptosis in vitro and in nude mouse xenografts. Oncol Rep 30: 1782-1792, 2013. 